\title{
Associations between allergic conditions and pediatric brain tumors in Neurofibromatosis Type 1
}

\author{
Bree Porcelli ${ }^{1}$, Nancy L. Zoellner, MPH ${ }^{2}$, Salmafatima S. Abadin, MPH $^{2}$, David H. Gutmann, \\ $\mathrm{MD}, \mathrm{PhD}^{1}$, and Kimberly J. Johnson, $\mathrm{MPH}, \mathrm{PhD}^{2}$ \\ ${ }^{1}$ Department of Neurology, Washington University School of Medicine, St. Louis, Missouri 63110 \\ ${ }^{2}$ Brown School, Washington University, St. Louis, Missouri 63130
}

\begin{abstract}
Background-Individuals with Neurofibromatosis type 1 (NF1) are at increased risk for pediatric brain tumors (PBTs), especially optic gliomas; however, factors influencing their development are largely unknown. Extensive research suggests that allergic conditions protect against brain tumors, particularly gliomas in individuals without NF1. In this large cross-sectional study, we employed two different data sources to evaluate evidence for the hypothesis that allergic conditions (allergies, asthma, and eczema) may protect against PBT development in individuals with NF1.
\end{abstract}

Methods-We used self- and parent/legal guardian reported questionnaire data from participants in the NF1 Patient Registry Initiative (NPRI, n=1660) born from 1933-2014 to ascertain allergic condition and PBT diagnosis histories. Medical records (MRs) of $629 \mathrm{NF} 1$ patients at a large medical center born from 1930-2012 were also reviewed for PBT and allergic condition diagnoses to evaluate additional evidence for our hypothesis. We used logistic regression to calculate odds ratios (ORs) and 95\% confidence intervals (CIs) for associations between allergic condition diagnoses and PBTs.

Results-Both data sources provided limited to no support for a protective effect of allergies or eczema on PBT development. Non-significant inverse associations between asthma and PBTs were observed (NPRI: OR=0.80, 95\% CI 0.55-1.17; MR: OR=0.71, 95\% CI 0.40-1.28) with stronger associations for optic gliomas specifically. Additionally, a significant inverse association was observed in an NPRI subgroup analysis where the reported asthma diagnosis age was younger than the reported PBT diagnosis age $(\mathrm{OR}=0.57$; 95\% CI $0.36-0.89)$.

Conclusion-Our study supports the hypothesis that asthma protects against PBT development in NF1.

\section{Keywords}

Neurofibromatosis Type 1; brain tumors; allergies; asthma; optic pathway gliomas

Corresponding author: Kimberly J. Johnson, MPH, PhD, Brown School, 237 Goldfarb Hall, Campus Box 1196, Washington University in St. Louis, One Brookings Drive, St. Louis, MO 63130, Phone: 1-314-935-9154, Fax: 1-314-935-8511, kijohnson@wustl.edu.

Conflict of Interest Statement: The authors declare that they have no conflict of interest to disclose. 


\section{INTRODUCTION}

Neurofibromatosis Type 1 (NF1) is an autosomal dominant disorder caused by germline mutations in the NF1 tumor suppressor gene [1] that affects an estimated 1 in 3000 individuals [2,3]. Individuals with NF1 have a variety of clinical manifestations, including pigmentary abnormalities (café-au-lait spots, skinfold freckling, and Lisch nodules), peripheral nerve sheath tumors (neurofibromas), bone defects, learning and behavioral deficits, and increased tumor susceptibility [4]. Despite having complete genetic penetrance, phenotypic expression of NF1 varies widely among affected individuals, even in individuals from the same family [5].

Children with NF1 are at high risk for pediatric brain tumors (PBTs), particularly optic pathway gliomas (OPGs) that are diagnosed in $~ 15-20 \%$ of children with this condition and can impair vision [6]. By comparison, $<0.1 \%$ of individuals < age 20 years will be diagnosed with a brain tumor [7]. Despite the high PBT incidence in the NF1 population, risk factors for their development are largely unknown. In addition to informing the underlying biology of PBTs, identification of factors that modulate risk is a critical component of developing PBT risk prediction models for this population and for developing prevention strategies. To date, some studies have indicated that individuals with African ancestry are at lower risk of PBT development than those with European ancestry [8-13] and two small studies have suggested that children harboring $N F 1$ gene mutations closer to its $5^{\prime}$ end are more likely to develop OPGs $[14,15]$.

Recent studies evaluating risk factors for gliomas in adults in the general population have emphasized the potential role of immune factors in their etiology, particularly that allergic conditions may protect against their development [16-19] through enhanced immune surveillance [20]. There is also evidence indicating that allergic conditions may confer protection against the development of PBTs [21]. However, associations between allergic conditions and PBTs in the NF1 population specifically have not previously been investigated.

In the present study, our goal was to evaluate evidence for the hypothesis that individuals with NF1 who have allergic condition diagnoses (allergies, eczema, asthma) are at lower risk of PBTs and OPGs using data collected through two different sources: 1) the web-based Neurofibromatosis Patient Registry Initiative (NPRI), and 2) patient medical records (MRs) from a large tertiary referral medical center. The results of this study may inform the etiology of PBTs in individuals with NF1.

\section{METHODS}

\section{Study populations}

This study included participants from the NPRI (https://nf1 registry.wustl.edu), an online registry for individuals with NF1, who enrolled between 5/17/2011 and 4/3/2015 and provided information about past brain tumor diagnoses (1660 of 2147 participants were included). Registry methodological details have been previously published [21-23]. Briefly, adults and minors with self- or parent/legal guardian-identified NF1 are eligible to 
participate in the NPRI. Following online consent, individuals $\geq 18$ years at the time of registration or a parent/legal guardian of individuals $<18$ years of age are instructed to complete a 30-45 minute questionnaire (with specific versions for adults and minors) that inquires about demographic, clinical (including NF1 clinical signs), and psychosocial history. Participant data are stored at Washington University in St. Louis behind a secure firewall. Link Plus (http://www.cdc.gov/cancer/npcr/tools/registryplus/lp.htm), a probabilistic record linkage program created by the Center for Disease Control and Prevention's Division of Cancer Prevention and Control, was used to identify individuals who had multiple questionnaire records in the NPRI with only data obtained from the most complete questionnaire used in the analysis being included in the analytic dataset.

This study also included MR data from subjects who were ascertained from the Clinical Investigation Data Exploration Repository (CIDER), a comprehensive inpatient and outpatient research patient data warehouse created by the Washington University Center for Biomedical Informatics (http://cbmi.wustl.edu/?q=project/cider). We queried CIDER for International Classification of Diseases Ninth Revision (ICD-9) codes 237.70 or 237.71 for Neurofibromatosis to identify an initial set of 820 individuals with NF ICD-9 codes documented in their medical records from 7/1/1997 to 6/1/2014. Of these, 634 unique cases were verified through medical record review for the NF1 NIH diagnostic criteria [24] as described previously [25]. Patient records that included physician notes (history and physical), patient questionnaires, anesthesia records, outpatient surgery and prehospitalization forms, imaging records, administrative notes, and allergy status notes from inpatient records were examined for reported brain tumor and allergy/asthma diagnoses along with age of diagnoses. A RedCap database (http://project-redcap.org/) was constructed to allow for abstraction of relevant analytic variables from patient records. Only individuals with MRs containing sufficient information to determine that their brain tumor diagnosis occurred $<18$ years of age were included ( $\mathrm{n}=629$ of 634 ). All procedures performed in studies involving human participants were in accordance with the ethical standards of the institutional research committee and with the 1964 Helsinki declaration and its later amendments or comparable ethical standards. For the MR study, formal consent is not required. The Institutional Review Board at Washington University in St. Louis approved all protocols for this study.

\section{Variable measurement}

NPRI subjects-Demographic information obtained from the questionnaire was used to construct variables for sex (male, female), race (Black, White, Asian, Other/Unknown), ethnicity (Hispanic, non-Hispanic), birth year category (<1969, 1969-1984, 1985-2001, $>2001$ ), residency (U.S., non-U.S.), having an NF-specialist (yes, no), and highest household education level ( $\leq$ to high school or a high school/high school equivalent degree, some college/no degree or a technical degree, zto a college degree). Brain tumor diagnoses were ascertained on the minor and adult questionnaires through the question "[Has the participant/ Have you] ever been diagnosed with a brain tumor?' with possible responses of 'Yes', 'No', and 'Don't Know'. If the respondent selected 'Yes', they were further prompted to specify the diagnosis age through the question "How old [was the participant/were you] when the brain tumor was diagnosed?". PBTs were defined based on responses to these two questions 
as those diagnosed $<18$ years of age. If the age at diagnosis was missing, a brain tumor diagnosis occurring during childhood was inferred if the subject was reported to be $<18$ years old at the time of NPRI participation. The NPRI questionnaire did not specifically ask about specific brain tumor subtypes, however we were able to determine OPG diagnoses for a subset of respondents. For these individuals, we confirmed brain tumor presence through medical records that were obtained or through a write-in response that indicated a subtype on the NPRI questionnaire to a question that asked about other cancer/tumor diagnoses in the participant.

Asthma, allergy, and eczema history were each assessed through the following question format "Has a doctor or other health professional EVER told you that [the participant has/you have] [asthma/allergies/eczema]?" In addition, for each of these conditions, respondents were asked the age at which they were told that they had or the minor participant had the condition. Allergy types were further identified through the following question format "[Has the participant/Have you] EVER had allergic reactions (rash, itchy/ watery eyes, itchiness, congestion, diarrhea, vomiting or difficulty breathing) to any of the following (please select all that apply)?" Respondents could check boxes for animals (dogs, cats, rabbits, birds, horses, etc.), eggs, dairy products, shellfish, wheat, peanuts or peanut butter, other nuts, house dust, mold or mildew, trees, grass, weeds, pollen, poison oak/ivy, stinging or biting insects, soy, none, and other (specify: ). Individuals were classified as having food allergies if they indicated one of the food allergies listed above through checking a box or through foods that were specified through a write-in response, otherwise they were classified as not having a food allergy. We also created a variable to assess seasonal allergies and general allergic rhinitis. If the participant checked any of the boxes for pollen, trees, grass, weeds, or reported seasonal or hay allergies through a write-in response then they were classified as having seasonal allergies, otherwise they were classified as not having seasonal allergies. Our allergic rhinitis definition included seasonal allergies and reported allergies to mold or mildew, house dust, and animals either through checkboxes or write-in responses. If none of these allergy types were specified, the participant was classified as not having allergic rhinitis.

MR subjects-Demographic and clinical information abstracted from MRs was used to construct variables for sex, race/ethnicity (White, Black, Asian, Hispanic, Unknown, Other), and birth year category $(<1969,1969-1984,1985-2001,>2001)$. Clinical information on brain tumor history (subtype and age first detected), allergy history, other allergic condition history (eczema, psoriasis, none), and asthma history were abstracted to construct analytic variables as described below. As for the NPRI, PBTs ascertained from MRs were defined as those reported to be diagnosed at $<18$ years of age. Individuals were classified as having any allergy if there was a report of any allergy in their record excluding medication allergies. Individuals were classified as having seasonal allergies if the MR specifically documented "seasonal" allergies, "hay fever", or there was a report of allergies to plants (e.g. plants, trees, ragweed, grass, pollen). For allergic rhinitis, individuals were classified as having allergic rhinitis if the MR indicated that they had any of the seasonal allergies noted above or if there was a report of animal, dust, and/or mold allergies. Individuals were categorized as having a food allergy, eczema, and asthma if their MR indicated the presence of any of these 
conditions. If an individual's MR did not contain any documentation of specific allergic conditions as noted above, they were classified as not having the condition.

\section{Statistical analyses}

We used Statistical Analysis Software (SAS) version 9.3 (SAS Institute, Cary, NC) to conduct all analyses. Multivariate logistic regression was used to calculate odds ratios (ORs) and 95\% confidence intervals (CIs) for associations between allergic conditions and PBTs after adjusting for covariates. We also conducted subgroup analyses that accounted for the temporal order of allergic condition and PBT diagnoses to evaluate whether the data were consistent with the hypothesis that allergic conditions protect against PBT development. For these analyses, we first identified PBT cases whose brain tumor diagnosis age was reported to have occurred at a younger age than their allergic condition diagnosis age. We then matched these PBT cases to non-cases who had a reported birth year and allergic condition diagnosis age within three years of age of the PBT case ages for these variables using the gmatch algorithm [26]. The matched sets of PBT cases and non-cases were then excluded from the analytic dataset. Model fit was assessed by the Hosmer and Lemeshow goodnessof-fit test [27] using the lackfit option in SAS. Tests for interaction were performed by including the cross product of the allergic condition variable and the subgroup defining variable (i.e. age group and household education level) in logistic regression models to determine if there was evidence that associations between allergic conditions and PBTs were significantly different between subgroups. All statistical tests were two sided with results considered statistically significant at $\mathrm{p}<0.05$.

\section{RESULTS}

A total of 1660 and 629 individuals with data obtained from the NPRI and MRs, respectively, were included in analyses. One hundred thirty eight subjects were in both datasets. The median reported PBT diagnosis age was 5 years for both NPRI and MR subjects. For the NPRI, individuals with reported PBTs included a higher percentage of males than those without reported PBTs, while for MR individuals there was higher percentage of females among those with PBTs. For both data sources, a higher frequency of individuals with PBT diagnoses than without PBT diagnoses had reported White race with no marked difference in the distribution of Hispanic ethnicity by PBT diagnosis status. Cases were more likely to be born in recent birth years than non-cases for both data sources. For the NPRI, the distribution of highest household education level and U.S. residency was similar between cases and non-cases. Finally, more NPRI PBT cases had a reported NF specialist than non-cases (Table 1).

In models adjusted for birth year category and sex, we observed no significant associations between any reported allergy (NPRI: OR=1.34, 95\% CI 0.99-1.83; MR: OR=0.82, 95\% CI $0.47-1.41$ ), allergic rhinitis (NPRI: OR $=1.05,95 \%$ CI $0.77-1.44$; MR: OR $=1.13,95 \%$ CI 0.61-2.09), or seasonal allergies (NPRI: OR=1.11, 95\% CI 0.79-1.55; MR: OR=1.03, 95\% CI 0.55-1.93) and PBTs. Similarly, for food allergies and eczema, we did not find evidence to support a protective effect with ORs of 1.25 (95\% CI 0.85-1.82) and 1.07 (95\% CI 0.771.50) respectively based on NPRI data only where numbers were sufficient for analysis. In 
support of our hypothesis, there were consistent non-significant inverse associations between asthma history and PBTs with ORs of 0.80 (95\% CI 0.55-1.17) and 0.71 (95\% CI 0.40 1.28) for NPRI and MR subjects respectively (Table 2). Of note, models were also run that included race and U.S. residency as covariates; however the results were not materially different and therefore only birth year category and sex were included in final models in Table 2. We also examined whether associations varied by household education level and age category ( $<20$ vs. $\geq 20$ years) for the NPRI where data on education was available and found no significant interactions (data not shown).

Although numbers were small, additional analyses indicated that the inverse association between asthma and PBTs was stronger for OPGs specifically in both data sources after adjusting for birth year category and sex (NPRI: OR=0.64, 95\% CI $0.32-1.28, \mathrm{n}=10$ and 60 PBT cases with and without asthma, respectively; MR: OR=0.64, 95\% CI 0.33-1.25, n=12 and 77 PBT cases with and without asthma, respectively) (table not shown).

Because allergic conditions would only be expected to protect against PBT development when present prior to its development, we conducted subgroup analyses using NPRI data for any allergy, asthma, and eczema where cases (and matched non-cases) were excluded from the analysis if their allergic condition diagnosis was reported to have occurred at the same or older age than their PBT diagnosis. For any allergy, asthma, and eczema, 51, 14, and 23 matched case and non-case sets were excluded, respectively. All associations became inverse with ORs of 0.85 for any allergy (95\% CI 0.60-1.21), 0.81 for eczema (95\% CI 0.56-1.19), and 0.57 for asthma (95\% CI 0.36-0.89) (Table 3). Of note, we did not conduct a similar analysis for MR subjects, because the data on diagnoses ages for allergic conditions was based on the record date that the allergic condition was recorded with the age at initial diagnosis often not recorded in the MR.

Finally, we conducted analyses stratified by age group ( $<18$ vs. $\geq 18$ years) at the time the questionnaire was last updated for NPRI participants to address whether associations varied by age of the participants. The goal of this analysis was to evaluate potential differences in associations by age group that could be due to differential reporting of allergic condition and PBT diagnoses by either parents/legal guardians of minor participants versus older participants. There were no significant differences in associations by age group with $\mathrm{p}$ values for interaction of $0.40,0.89$, and 0.46 for allergies, eczema, and asthma respectively. However, we note that the association between asthma and PBTs was stronger in the younger $(\mathrm{OR}=0.46,95 \% \mathrm{CI}=0.26-0.79)$ than in the older $(\mathrm{OR}=0.83$; 95\% CI 0.39-1.77) age group (Table 3).

\section{DISCUSSION}

There is intense interest in the role of the immune system in cancer development and treatment. To our knowledge, this is the first study in the NF1 population to evaluate a potential role for allergic conditions in PBT etiology. The strongest evidence in support of our hypothesis was the observed inverse association between asthma and PBTs, especially when only individuals whose asthma diagnosis was reported to occur before their PBT diagnosis were included in the analysis. 
Although this is the first study on this topic in the NF1 population, there is substantial evidence that allergic conditions protect against adult gliomas from previous reports in general population samples. Three meta-analyses of allergic history and adult glioma demonstrate a $\sim 20-40 \%$ risk reduction in individuals with asthma, eczema, hay fever or allergy histories [16-18]. In children, this question has been less well investigated. One study reported a significant inverse association between asthma and CNS tumors (OR=0.75; 95\% CI 0.58-0.97) but not eczema (OR=0.94; 95\% CI 0.74-1.18), with the strongest association for individuals who had both asthma and eczema ( $\mathrm{OR}=0.48$; 95\% CI $=0.28$ 0.81 ). However, this study reported that the effect was strongest for medulloblastoma/ primitive neural ectodermal tumors, which are tumors of non-glial origin not typically found in children with NF1 [28]. Similarly, another study found an inverse association between asthma and PBTs, with a magnitude similar to that found in our study (OR=0.55; 95\% CI $0.33-0.93)$. These authors reported non-significant associations for eczema ( $\mathrm{OR}=0.52 ; 95 \%$ CI 0.71-1.57) and for both asthma and eczema (OR=0.76; 95\% CI 0.18-3.2) [29]. Finally, a recent study reported no evidence for an association between ever having asthma and all PBTs, including glioma. The authors also examined current and past asthma diagnoses and found no significant association with PBTs overall or specific subtypes. However, this study involved children with brain tumors that were diagnosed between the ages of 7-19 years, which may have a different etiology than those with younger ages of onset included in the former studies [30].

Although the mechanisms underlying a protective effect of asthma on brain tumor development are not well understood, one of the leading hypotheses for inverse associations between atopic conditions and cancer is enhanced immune surveillance in individuals with these conditions [20]. In this regard, the immune system in individuals with allergic conditions is thought to recognize and eliminate premalignant cells more effectively than the immune system in individuals without these conditions. Many investigators have focused on Immunoglobulin $\mathrm{E}$ ( $\mathrm{IgE}$ ), an allergy biomarker, to evaluate evidence for a protective role of allergic conditions in cancer development; however, results from studies have been somewhat mixed [31,32]. This may partially be due to difficulties in obtaining a premalignancy IgE measurement in cancer cases, resulting in uncertainties about cause and effect relationships. However, in one study that obtained samples that were taken $>20$ years prior to the brain tumor diagnosis, an inverse association was reported [31]. Additionally, evidence from $N f 1$ genetically-engineered mouse studies has demonstrated that immune system-like cells (microglia) are necessary for both optic glioma formation and continued growth [33-36]. The relationship between these brain microglia and those involved in atopic or asthmatic conditions is unclear.

As with all observational studies, our study has strengths and limitations. The major strengths of this study are its size and the use of two different data sources to examine associations between allergic conditions and PBTs. We also note some limitations of our study. First, both the NPRI and MR data were collected from non-population based samples of individuals with NF1 raising the possibility of selection bias. Obtaining, a large population-based sample of individuals with NF1 to examine whether allergic conditions are associated with PBT development is not currently feasible in most countries, with the possible exception of the Nordic countries that health collect information through 
population-based registries. Therefore, our results should be considered within the context of this limitation and in need of further validation.

Second, since data on allergic condition and PBT diagnoses are cross-sectional in nature, the temporal order of their occurrence is difficult to discern for some individuals, particularly for MR subjects. We were able to address this for the NPRI subjects in our analyses that controlled for the temporal order of allergic condition and PBT diagnoses, finding stronger evidence for a protective effect on PBT risk for asthma when asthma was reported to have been diagnosed prior to the PBT diagnosis.

Third, medical condition information was participant or parent/legal guardian reported for the NPRI and to some extent for MR subjects where patient medical history is sometimes captured from the patient or their parent/legal guardian rather than from physical examination or medical tests. Of note, our previous studies suggest that NF1 [23] and PBT diagnoses are reported with good accuracy for NPRI participants [13]. For allergic condition diagnoses, participant/parent legal guardian reported data could result in misclassification of subjects with respect to allergic condition diagnoses if conditions are not actually present in the participant or they are present and not reported. Reporting accuracy of childhood diagnoses may be a particular problem in adults who may not recall correctly or be aware of past medical diagnoses. The association between asthma and PBTs was stronger in younger children, which may reflect more accurate reporting of recent asthma diagnoses and age of diagnosis by caregivers than in adults whose childhood asthma diagnoses may have occurred years ago. For asthma, where we found the strongest inverse associations, reporting accuracy has been noted to have high validity in general for prevalent asthma in one study that compared self-reported to physician diagnosed asthma [37].

Finally, we note that the MRs were often unclear with respect to allergy type and minor allergies may not have been reported in the MRs that were ascertained for this study. Underreporting seems less likely to be an issue for asthma where the condition is often serious. More accurate reporting for asthma vs. other allergic conditions may explain why the prevalence of asthma that was calculated from data captured from MRs and the NPRI questionnaire was more similar between these sources than for other allergic conditions.

In conclusion, we found evidence in support of our hypothesis for a protective effect of asthma but not other allergic conditions on PBT development in individuals with NF1. Replication across additional NF1 study populations will be necessary before making any causal inferences about the role of asthma in PBT development in NF1. Should these results be replicated, further investigations should focus on the mechanistic underpinnings of this association. This work could ultimately impact clinical risk prediction models for children with NF1.

\section{Acknowledgments}

Supported by Alex's Lemonade Stand Foundation, the American Cancer Society Institutional Research Grant (IRG-58-010-55), and the National Institutes of Health (Clinical and Translational Science Q6 Award UL1 TR000448). 


\section{References}

1. Marchuk DA, Saulino AM, Tavakkol R, et al. cDNA cloning of the type 1 neurofibromatosis gene: complete sequence of the NF1 gene product. Genomics. 1991; 11(4):931-40 D. DOI 0888-7543(91)90017-9 [pii]. [PubMed: 1783401]

2. Friedman JM. Epidemiology of neurofibromatosis type 1. American journal of medical genetics. 1999; 89(1):1-6. [PubMed: 10469430]

3. Rasmussen SA, Friedman JM. NF1 gene and neurofibromatosis 1. American journal of epidemiology. 2000; 151(1):33-40. [PubMed: 10625171]

4. Lynch TM, Gutmann DH. Neurofibromatosis 1. Neurol Clin. 2002; 20(3):841-65. [PubMed: 12432832]

5. Viskochil D. Genetics of neurofibromatosis 1 and the NF1 gene. Journal of child neurology. 2002; 17(8):562-70. discussion 71-2, 646-51. [PubMed: 12403554]

6. Albers AC, Gutmann DH. Gliomas in patients with neurofibromatosis type 1. Expert review of neurotherapeutics. 2009; 9(4):535-9. DOI: 10.1586/ern.09.4 [PubMed: 19344304]

7. American Cancer Society. Cancer Facts \& Figures 2014. Atlanta: American Cancer Society; 2014.

8. Blazo MA, Lewis RA, Chintagumpala MM, Frazier M, McCluggage C, Plon SE. Outcomes of systematic screening for optic pathway tumors in children with Neurofibromatosis Type 1. American journal of medical genetics Part A. 2004; 127A(3):224-9. DOI: 10.1002/ajmg.a.20650 [PubMed: 15150770]

9. Kaste SC, Pivnick EK. Bony orbital morphology in neurofibromatosis type 1 (NF1). Journal of medical genetics. 1998; 35(8):628-31. [PubMed: 9719366]

10. King A, Listernick R, Charrow J, Piersall L, Gutmann DH. Optic pathway gliomas in neurofibromatosis type 1: the effect of presenting symptoms on outcome. American journal of medical genetics Part A. 2003; 122A(2):95-9. DOI: 10.1002/ajmg.a.20211 [PubMed: 12955759]

11. Pletcher BAMM, Frohman LP, Wagner RS, DeRespinis P, Kamin S, Chatkupt S. Confirmation of decreased risk of optic glioma in African Americans with NF type 1 (Abstract). American Journal of Human Genetics. 1996; 59(4):A101.

12. Saal HMSE, Lovell AM, Ball W, Egelhoff J, Koch B, Samango Sprouse CA, Rosenbaum KN, Stern HJ, Tiffi CJ, Vezina LG. Racial differences in the prevalence of optic nerve gliomas in neurofibromatosis type 1. American Journal of Human Genetics. 1995; 57(4):280.

13. Abadin SS, Zoellner NL, Schaeffer M, Porcelli B, Gutmann DH, Johnson KJ. Racial/Ethnic Differences in Pediatric Brain Tumor Diagnoses in Patients with Neurofibromatosis Type 1. The Journal of pediatrics. 2015; 167(3):613-20 e2. DOI: 10.1016/j.jpeds.2015.04.076 [PubMed: 26028287]

14. Sharif S, Upadhyaya M, Ferner R, et al. A molecular analysis of individuals with neurofibromatosis type 1 (NF1) and optic pathway gliomas (OPGs), and an assessment of genotype-phenotype correlations. Journal of medical genetics. 2011; 48(4):256-60. DOI: 10.1136/ jmg.2010.081760 [PubMed: 21278392]

15. Bolcekova A, Nemethova M, Zatkova A, et al. Clustering of mutations in the $5^{\prime}$ tertile of the NF1 gene in Slovakia patients with optic pathway glioma. Neoplasma. 2013; 60(6):655-65. DOI: 10.4149/neo_2013_084 [PubMed: 23906300]

16. Zhao H, Cai W, Su S, Zhi D, Lu J, Liu S. Allergic conditions reduce the risk of glioma: a metaanalysis based on 128,936 subjects. Tumour biology: the journal of the International Society for Oncodevelopmental Biology and Medicine. 2014; 35(4):3875-80. DOI: 10.1007/ s13277-013-1514-4 [PubMed: 24347487]

17. Chen C, Xu T, Chen J, et al. Allergy and risk of glioma: a meta-analysis. European journal of neurology: the official journal of the European Federation of Neurological Societies. 2011; 18(3): 387-95. DOI: 10.1111/j.1468-1331.2010.03187.x

18. Linos E, Raine T, Alonso A, Michaud D. Atopy and risk of brain tumors: a meta-analysis. Journal of the National Cancer Institute. 2007; 99(20):1544-50. DOI: 10.1093/jnci/djm170 [PubMed: 17925535] 
19. Turner MC, Krewski D, Armstrong BK, et al. Allergy and brain tumors in the INTERPHONE study: pooled results from Australia, Canada, France, Israel, and New Zealand. Cancer causes \& control: CCC. 2013; 24(5):949-60. DOI: 10.1007/s10552-013-0171-7 [PubMed: 23443320]

20. Sherman PW, Holland E, Sherman JS. Allergies: their role in cancer prevention. The Quarterly review of biology. 2008; 83(4):339-62. [PubMed: 19143335]

21. Johnson KJ, Cullen J, Barnholtz-Sloan JS, et al. Childhood brain tumor epidemiology: a brain tumor epidemiology consortium review. Cancer epidemiology, biomarkers \& prevention: a publication of the American Association for Cancer Research, cosponsored by the American Society of Preventive Oncology. 2014; 23(12):2716-36. DOI: 10.1158/1055-9965.EPI-14-0207

22. Johnson KJ, Hussain I, Williams K, Santens R, Mueller NL, Gutmann DH. Development of an international internet-based neurofibromatosis Type 1 patient registry. Contemporary clinical trials. 2013; 34(2):305-11. DOI: 10.1016/j.cct.2012.12.002 [PubMed: 23246715]

23. Sharkey EK, Zoellner NL, Abadin S, Gutmann DH, Johnson KJ. Validity of participant-reported diagnoses in an online patient registry: A report from the NF1 Patient Registry Initiative. Contemporary clinical trials. 2014; 40C:212-7. DOI: 10.1016/j.cct.2014.12.006

24. Neurofibromatosis. Conference statement. National Institutes of Health Consensus Development Conference. Archives of neurology. 1988; 45(5):575-8. [PubMed: 3128965]

25. Ostendorf AP, Gutmann DH, Weisenberg JL. Epilepsy in individuals with neurofibromatosis type 1. Epilepsia. 2013; 54(10):1810-4. DOI: 10.1111/epi.12348 [PubMed: 24032542]

26. Bergstralh EKJ. gmatch: Computerized matching of cases to controls using the greedy matching algorithm with a fixed number of controls per case. 2003 May 23. vol.

27. Hosmer, DW., Jr; Lemeshow, S. Applied Logistic Regression. Second. New York: John Wiley \& Sons; 2000 .

28. Harding NJ, Birch JM, Hepworth SJ, McKinney PA. Atopic dysfunction and risk of central nervous system tumours in children. European journal of cancer. 2008; 44(1):92-9. DOI: 10.1016/ j.ejca.2007.10.007 [PubMed: 18042376]

29. Roncarolo F, Infante-Rivard C. Asthma and risk of brain cancer in children. Cancer causes \& control: CCC. 2012; 23(4):617-23. DOI: 10.1007/s10552-012-9928-7 [PubMed: 22367725]

30. Shu X, Prochazka M, Lannering B, et al. Atopic conditions and brain tumor risk in children and adolescents-an international case-control study (CEFALO). Annals of oncology: official journal of the European Society for Medical Oncology/ESMO. 2014; 25(4):902-8. DOI: 10.1093/annonc/ mdu048

31. Schwartzbaum J, Ding B, Johannesen TB, et al. Association between prediagnostic IgE levels and risk of glioma. Journal of the National Cancer Institute. 2012; 104(16):1251-9. DOI: 10.1093/jnci/ djs315 [PubMed: 22855780]

32. Turner MC. Epidemiology: allergy history, IgE, and cancer. Cancer immunology, immunotherapy: CII. 2012; 61(9):1493-510. DOI: 10.1007/s00262-011-1180-6 [PubMed: 22183126]

33. Daginakatte GC, Gutmann DH. Neurofibromatosis-1 (Nf1) heterozygous brain microglia elaborate paracrine factors that promote Nf1-deficient astrocyte and glioma growth. Human molecular genetics. 2007; 16(9):1098-112. DOI: 10.1093/hmg/ddm059 [PubMed: 17400655]

34. Daginakatte GC, Gianino SM, Zhao NW, Parsadanian AS, Gutmann DH. Increased c-Jun-NH2kinase signaling in neurofibromatosis-1 heterozygous microglia drives microglia activation and promotes optic glioma proliferation. Cancer research. 2008; 68(24):10358-66. DOI: 10.1158/0008-5472.CAN-08-2506 [PubMed: 19074905]

35. Simmons GW, Pong WW, Emnett RJ, et al. Neurofibromatosis-1 heterozygosity increases microglia in a spatially and temporally restricted pattern relevant to mouse optic glioma formation and growth. Journal of neuropathology and experimental neurology. 2011; 70(1):51-62. DOI: 10.1097/NEN.0b013e3182032d37 [PubMed: 21157378]

36. Pong WW, Higer SB, Gianino SM, Emnett RJ, Gutmann DH. Reduced microglial CX3CR1 expression delays neurofibromatosis-1 glioma formation. Annals of neurology. 2013; 73(2):303-8. DOI: 10.1002/ana.23813 [PubMed: 23424002]

37. Oksanen T, Kivimaki M, Pentti J, Virtanen M, Klaukka T, Vahtera J. Self-report as an indicator of incident disease. Annals of epidemiology. 2010; 20(7):547-54. DOI: 10.1016/j.annepidem. 2010.03.017 [PubMed: 20538198] 
Table 1

Characteristics of individuals with NF1 ascertained from the NPRI and MRs by pediatric brain tumor diagnosis history.

\begin{tabular}{|c|c|c|c|c|}
\hline \multirow[b]{2}{*}{ Characteristic } & \multicolumn{2}{|c|}{ NPRI (n=1660) } & \multicolumn{2}{|c|}{ MR $(n=629)$} \\
\hline & $\begin{array}{c}\text { PBT cases }(n=239) \\
N(\%)\end{array}$ & $\begin{array}{c}\text { Non-cases }(n=1418) \\
N(\%)\end{array}$ & $\begin{array}{c}\text { PBT cases }(\mathrm{n}=113) \\
\mathbf{N}(\%)\end{array}$ & $\begin{array}{c}\text { Non-cases }(\mathrm{n}=516) \\
\mathbf{N}(\%)\end{array}$ \\
\hline \multicolumn{5}{|l|}{$\operatorname{Sex}^{a}$} \\
\hline Male & $104(43.5)$ & $520(36.7)$ & $57(50.4)$ & $275(53.3)$ \\
\hline Female & $135(56.5)$ & $898(63.3)$ & $56(49.6)$ & $241(46.7)$ \\
\hline \multicolumn{5}{|l|}{ Race $^{a}$} \\
\hline White & $211(88.3)$ & 1108 (78.0) & 95 (85.6) & $327(64.0)$ \\
\hline Black & $0(0.0)$ & $82(5.8)$ & $5(4.5)$ & $122(23.9)$ \\
\hline Asian & $1(0.4)$ & $70(4.9)$ & $1(0.9)$ & $4(0.8)$ \\
\hline Other/Unknown & $27(11.3)$ & $161(11.3)$ & $10(9.0)$ & $58(11.4)$ \\
\hline \multicolumn{5}{|l|}{ Ethnicity $^{a}$} \\
\hline Hispanic & $20(8.5)$ & $126(9.0)$ & $111(98.2)$ & $511(99.0)$ \\
\hline Non-Hispanic & $216(91.5)$ & 1269 (91.0) & $2(1.8)$ & $5(1.0)$ \\
\hline \multicolumn{5}{|l|}{ Birth year ${ }^{a}$} \\
\hline$<1969$ & $6(2.5)$ & $422(29.9)$ & $2(1.8)$ & $93(18.0)$ \\
\hline 1969-1984 & $19(8.0)$ & $384(27.2)$ & $4(3.5)$ & 95 (18.4) \\
\hline 1985-2001 & $89(37.2)$ & $293(20.8)$ & $51(45.1)$ & $152(29.5)$ \\
\hline$>2001$ & $125(52.3)$ & $313(22.2)$ & $56(49.6)$ & $176(34.1)$ \\
\hline \multicolumn{5}{|l|}{ Highest household education level ${ }^{a}$} \\
\hline$₫ \mathrm{HS}, \mathrm{HS} / \mathrm{HS}$ equivalent degree & $54(22.8)$ & $363(25.6)$ & NR & NR \\
\hline Some college/no degree or a technical degree & $94(39.7)$ & $578(40.8)$ & NR & NR \\
\hline$\geq$ College degree & 89 (37.6) & $476(33.6)$ & NR & NR \\
\hline \multicolumn{5}{|l|}{ Residency $^{a}$} \\
\hline US & $187(79.6)$ & $1080(77.3)$ & NA & NA \\
\hline Non-US & $48(20.4)$ & $318(22.8)$ & NA & NA \\
\hline \multicolumn{5}{|l|}{ Reported having an NF specialist } \\
\hline Yes & $139(58.2)$ & $494(34.8)$ & NA & NA \\
\hline No & $100(41.8)$ & $927(65.2)$ & NA & NA \\
\hline
\end{tabular}

$\mathrm{NR}=$ Not reported in medical records; HS=high school; NA=Not applicable/available

a Missing data: sex (NPRI, $n=3$ ), race (MR, $n=7$ ), ethnicity (NPRI, $n=29)$, birth year (NPRI, $n=9)$, highest household education level ( $\mathrm{n}=6$ ), residency (NPRI, $\mathrm{n}=27)$ 


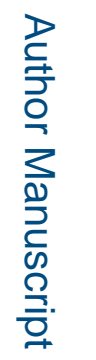

ᄅ⿳亠口冋.

롤

\begin{tabular}{|c|c|c|c|c|c|c|c|c|c|c|c|c|c|c|c|c|c|c|c|c|}
\hline $\begin{array}{l}\dot{1} \\
z \\
\text { Z }\end{array}$ & & 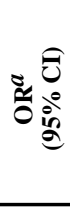 & & 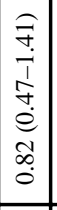 & $\begin{array}{l}\dot{\ddot{\Xi}} \\
\simeq\end{array}$ & & $\begin{array}{l}\hat{a} \\
i \\
\frac{1}{0} \\
e \\
0 \\
= \\
=\end{array}$ & 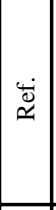 & & 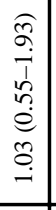 & 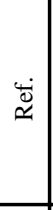 & & 之 & 合 & & 之 & 文 & & 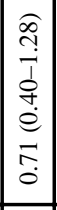 & 崩 \\
\hline$\frac{a}{0}$ & 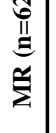 & 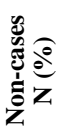 & & $\begin{array}{l}\widehat{\infty} \\
\stackrel{5}{\vdots} \\
\sigma \\
\sigma\end{array}$ & 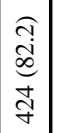 & & $\begin{array}{l}\hat{\partial} \\
\stackrel{\partial}{0} \\
\stackrel{\circ}{n}\end{array}$ & 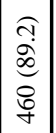 & & $\begin{array}{l}\hat{\sigma} \\
\stackrel{\rho}{0} \\
\text { in }\end{array}$ & 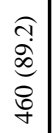 & & 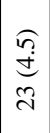 & 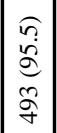 & & $\begin{array}{l}\underset{n}{+} \\
\underset{\sim}{+}\end{array}$ & 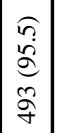 & & $\begin{array}{l}0 \\
\stackrel{0}{0} \\
\stackrel{0}{\Xi} \\
\stackrel{0}{0}\end{array}$ & $\begin{array}{l}\hat{f} \\
\dot{0} \\
\frac{0}{b} \\
\xi\end{array}$ \\
\hline $\begin{array}{l}. \Xi \\
. \Xi \\
0 \\
\varnothing \\
0\end{array}$ & & 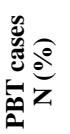 & & 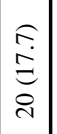 & $\begin{array}{c}\hat{\sigma} \\
\tilde{i} \\
\infty \\
\sigma \\
\sigma\end{array}$ & & 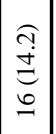 & $\begin{array}{c}0 \\
\alpha \\
\alpha \\
\alpha \\
\vdots \\
\sigma\end{array}$ & & $\begin{array}{l}\hat{\sigma} \\
\stackrel{ٍ}{n} \\
\stackrel{n}{n}\end{array}$ & $\begin{array}{l}\hat{F} \\
\dot{\delta} \\
\infty \\
\infty \\
\sigma\end{array}$ & & $\stackrel{\widehat{\infty}}{\underset{N}{\infty}}$ & $\mid \begin{array}{c}\widehat{a} \\
\infty \\
\stackrel{0}{0} \\
\Xi \\
\Xi\end{array}$ & & $\begin{array}{l}\underset{\sigma}{0} \\
\stackrel{\sigma}{\sigma}\end{array}$ & $\begin{array}{l}n \\
0 \\
0 \\
0 \\
0 \\
0\end{array}$ & & 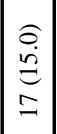 & 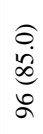 \\
\hline 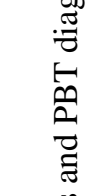 & $\widehat{\circ}$ & 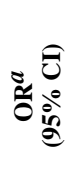 & & 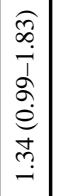 & $\stackrel{\vec{\varpi}}{\dddot{U}}$ & & 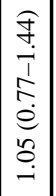 & $\begin{array}{l}\dot{\mathscr{\Xi}} \\
\propto\end{array}$ & & 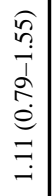 & $\ddot{\widetilde{\Xi}}$ & & 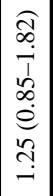 & $\ddot{\mathscr{U}}$ & & 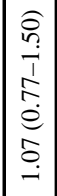 & $\dot{\mathscr{\Xi}}$ & & $\begin{array}{l}E \\
E \\
1 \\
n \\
n \\
0 \\
0 \\
0 \\
0 \\
0\end{array}$ & $\begin{array}{l}\dot{\widetilde{\Xi}} \\
\approx\end{array}$ \\
\hline 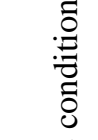 & 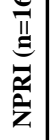 & 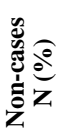 & & \begin{tabular}{|c|}
0 \\
$\dot{j}$ \\
$\dot{y}$ \\
$\infty$ \\
$i$ \\
$i$
\end{tabular} & 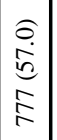 & & 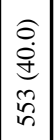 & 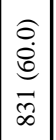 & & 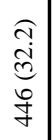 & $\begin{array}{l}\sigma \\
\infty \\
\tilde{\omega} \\
\infty \\
\sigma \\
\sigma\end{array}$ & & $\begin{array}{l}\underset{\hat{\sigma}}{\sigma} \\
\stackrel{\sigma}{0} \\
\approx \\
d\end{array}$ & $\mid \begin{array}{l}\widehat{\sigma} \\
\tilde{\infty} \\
\stackrel{0}{\Xi} \\
\Xi\end{array}$ & & $\begin{array}{l}\text { त̇ } \\
\text { ḋ } \\
\stackrel{\text { े }}{ }\end{array}$ & 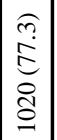 & & $\begin{array}{l}\stackrel{f}{d} \\
\underset{d}{d} \\
\underset{d}{d}\end{array}$ & 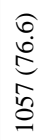 \\
\hline $\begin{array}{l}\frac{0}{\vec{U}} \\
\frac{\vec{\sigma}}{\bar{\Xi}} \\
\bar{\Xi}\end{array}$ & & 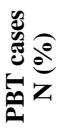 & & 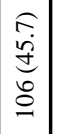 & $\begin{array}{l}\widehat{P} \\
\dot{0} \\
0 \\
0 \\
\end{array}$ & & $\begin{array}{l}\hat{\infty} \\
\stackrel{\vec{d}}{ } \\
\infty \\
\infty\end{array}$ & 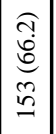 & & 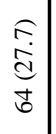 & $\begin{array}{l}\widehat{\widehat{n}} \\
\stackrel{5}{5} \\
\underline{b}\end{array}$ & & 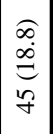 & 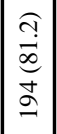 & & $\begin{array}{l}n \\
a \\
d \\
5\end{array}$ & $\begin{array}{l}n \\
\tilde{2} \\
8 \\
0 \\
0 \\
-\end{array}$ & & $\begin{array}{l}\tilde{\sigma} \\
\triangleq \\
\bar{\sigma} \\
\sigma\end{array}$ & 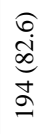 \\
\hline 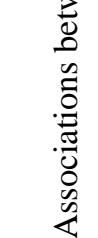 & & & 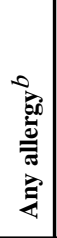 & $\lesssim$ & 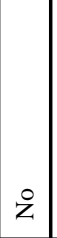 & 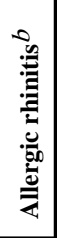 & 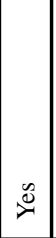 & $\dot{z}$ & 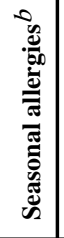 & $\stackrel{0}{0}$ & ż & 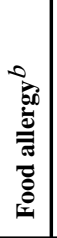 & $\stackrel{\mathscr{S}}{\sim}$ & z & 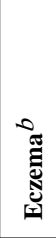 & $\stackrel{\mathscr{0}}{\circ}$ & z & 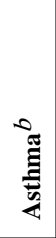 & $\succsim$ & $\stackrel{\circ}{z}$ \\
\hline
\end{tabular}

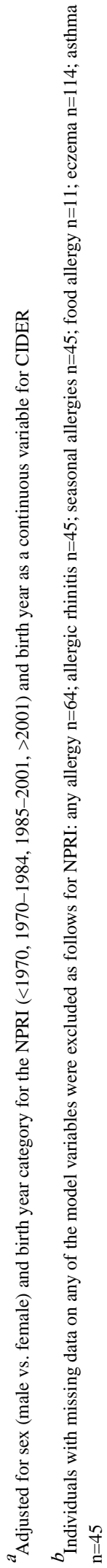

ᄅ⿳亠口冋.

年

 


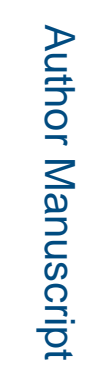

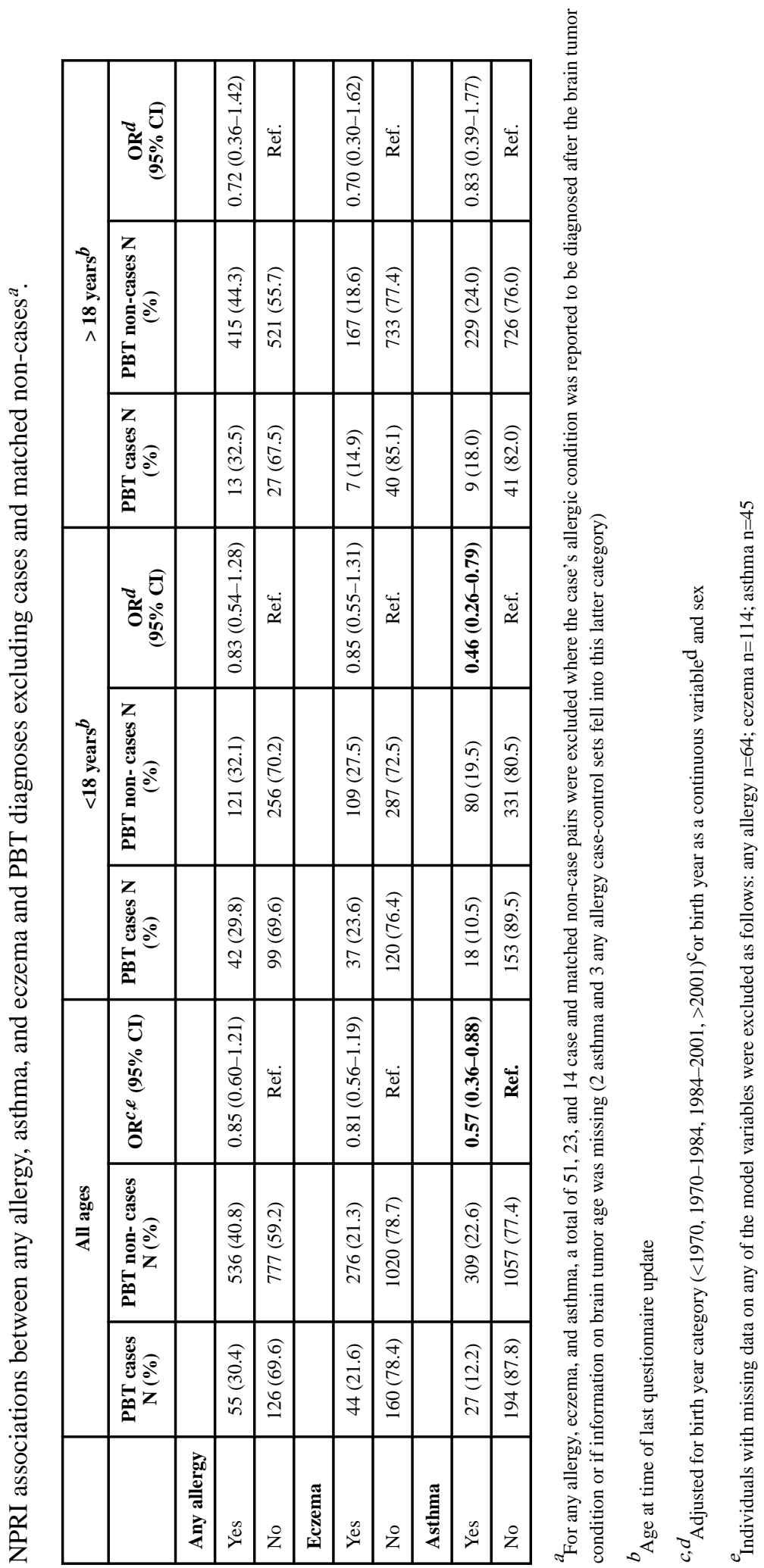

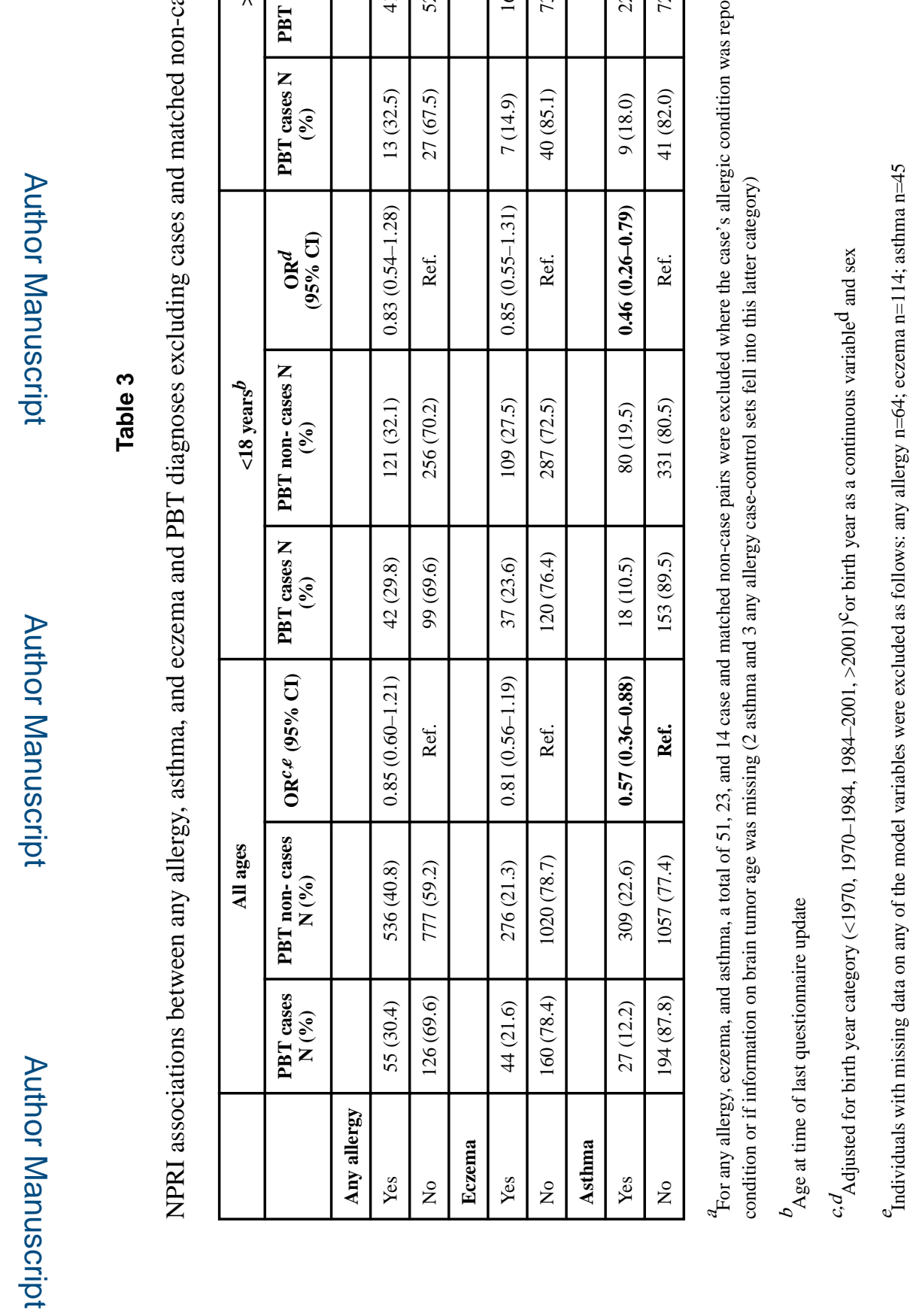

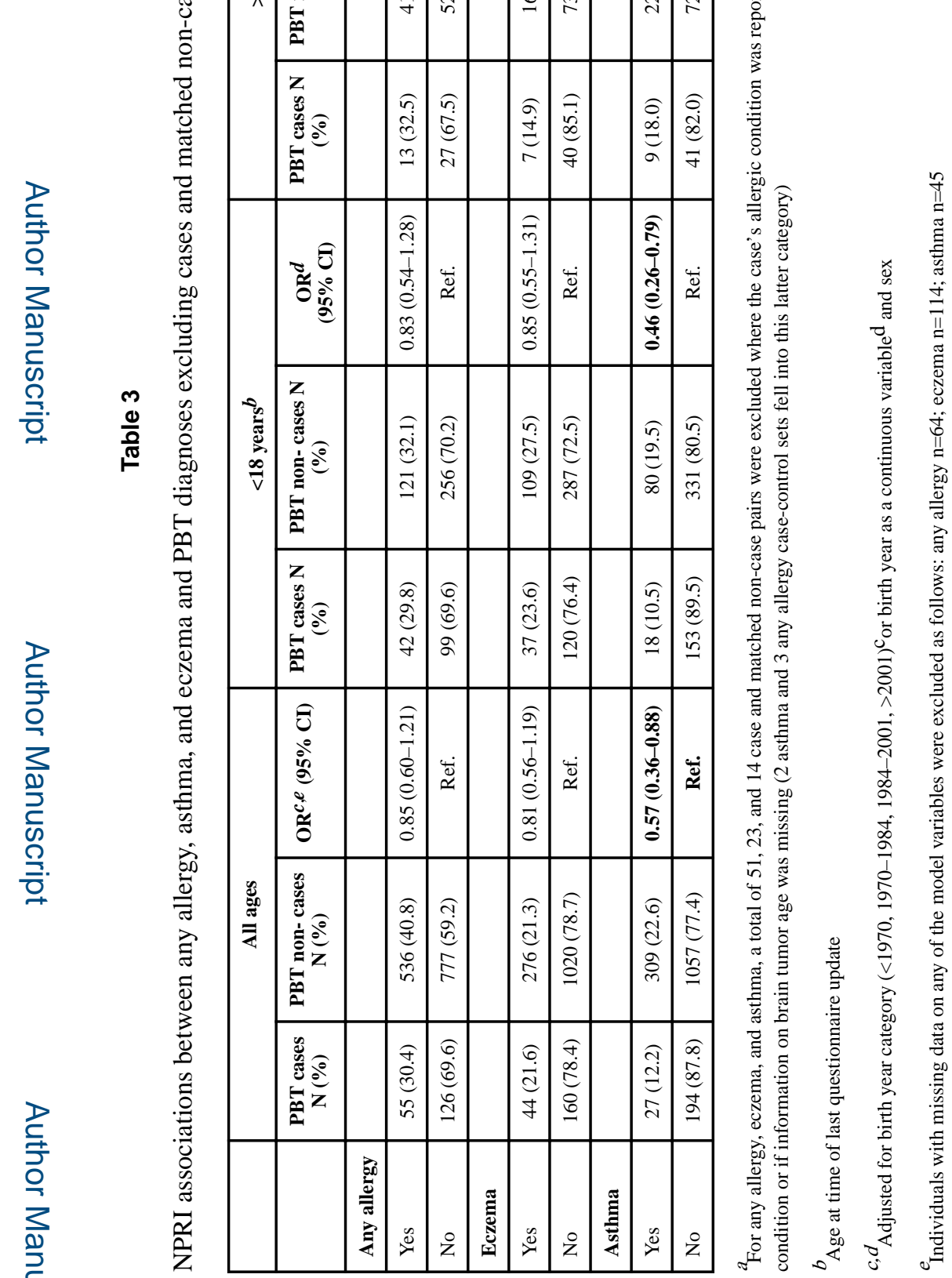

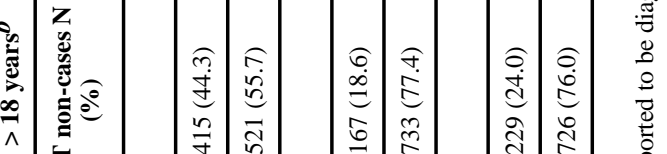

Fam Cancer. Author manuscript; available in PMC 2017 April 01. 